\title{
Homogeneous Graft Copolymerization of Vinyl Monomers onto Cellulose in a Dimethyl Sulfoxide- Paraformaldehyde Solvent System. I. Acrylonitrile and Methyl Methacrylate
}

\author{
Noboru NiSHIOKA and Kouichi KosAI \\ Faculty of Engineering, Osaka Electro-Communication \\ University, Neyagawa 572, Japan.
}

(Received July 31, 1981)

\begin{abstract}
Homogeneous graft copolymerization of acrylonitrile (AN) and of methyl methacrylate (MMA) onto cellulose was carried out in a dimethyl sulfoxide-paraformaldehyde solvent system. Ammonium persulfate (APS) and azobisisobutyronitrile (AIBN) were used as radical initiators. The optimum grafting condition for each monomer-initiator system was determined from experiments in which temperature and concentrations of initiator, monomer, and cellulose were varied. The grafting yield for the AN-APS system was higher than that for the MMA-APS system. The grafting onto cellulose hardly proceeded in the AN-AIBN system, but appreciably in the MMA-AIBN system. The grafting of MMA proceeded similarly either with AIBN or APS as the initiator. It was found that the chain transfer from PMMA homopolymer radicals to backbone cellulose occurred. The number of grafts per cellulose chain was found to be in the range from 0.5 to 3.9 .

KEY WORDS Homogeneous Grafting / Dimethyl Sulfoxide-Paraformaldehyde Solvent System / Acrylonitrile / Methyl Methacrylate / Grafting Percentage / Grafting Efficiency / Hydrolysis / Number of Grafts / Cellulose /
\end{abstract}

Graft copolymerization of vinyl monomers onto cellulose is usually carried out with fibrous cellulose. It is considered that graft copolymerization in such a heterogeneous system proceeds only partly and is affected markedly by the fine structure of the cellulose material used. ${ }^{1,2}$ Furthermore, use of different grafting methods gives rise to differences in composition, structure, etc., of the resulting products. $^{3-7}$

If grafting is carried out in a homogeneous system where cellulose dissolves molecularly, most of the complexities mentioned above may be circumvented. However, experimental data for such grafting are still meager, ${ }^{8,9}$ probably due to the lack of relevant solvent systems for cellulose.

Recently a number of non-aqueous solvents for cellulose have been found. ${ }^{10}$ Among them, dimethyl sulfoxide (DMSO) containing paraformaldehyde (PF) seems to be a promising candidate as a polymerization medium, because this mixture is a nondegrading solvent for cellulose ${ }^{11}$ and is expected to permit grafting to proceed without any side reactions.

The present study is concerned with grafting of acrylonitrile (AN) and of methyl methacrylate (MMA) onto cellulose in the DMSO-PF solvent system. Azobisisobutyronitrile (AIBN) as well as two peroxides, ammonium persulfate (APS) and benzoyl peroxide, were tested as initiators. Reaction temperature and concentrations of initiator, monomer, and cellulose were varied to find optimum conditions under which grafting onto cellulose would occur most effectively. Selected samples of the graft copolymers were analyzed for molecular weight of grafts and the number of grafts per cellulose backbone. Possible grafting mechanisms are discussed to account for the experimental results obtained. 


\section{EXPERIMENTAL}

Softwood pulp containing $93.5 \% \alpha$-cellulose and $3.6 \% \beta$-cellulose supplied by Fujibo Co. Ltd. was chosen as a cellulosic material. PF dried in a desiccator over $\mathrm{CaCl}_{2}$ and reagent grade DMSO were used without further purification. The monomers were purified by standard procedures.

Weighed amounts of well dried softwood pulp and PF powder ( $1: 1.2$ by weight) were dispersed in DMSO at room temperature. The mixture was heated with rapid stirring at $90-100^{\circ} \mathrm{C}$ until it became clear. Then the clear solution continued to be vigorously stirred at $130^{\circ} \mathrm{C}$ until formaldehyde ceased to bubble out. The clear solution obtained, designated as the DMSO solution, was used as the stock cellulose solution. The amount of cellulose in the DMSO solution was $2 \%$ by weight.

Appropriate amounts of the DMSO solution and an initiator were mixed in a three-necked flask and purified nitrogen was bubbled through the mixture for about $30 \mathrm{~min}$ at temperatures lower than $20^{\circ} \mathrm{C}$. Then, the required amount of a monomer was added. The polymerization was carried out at different fixed temperatures between 20 and $80^{\circ} \mathrm{C}$ and was finally terminated by adding hydroquinone. The polymerization mixture was poured into distilled water with vigorous stirring. The crude graft product was treated in a Soxhlet apparatus for more than $24 \mathrm{~h}$ to extract homopolymer with dimethylformamide (DMF) and acetone for poly(acrylonitrile) (PAN) and poly(methyl methacrylate) (PMMA), respectively. The infrared spectrum of each graft copolymer showed characteristic peaks for the corresponding homopolymer. This indicates that either PAN or PMMA was grafted onto the cellulose backbone.

The graft products were characterized by the following parameters:

$$
\begin{aligned}
& \text { weight conversion, WC }(\%) \\
& =(A-B) / C \times 100 \\
& \text { grafting percentage, GP }(\%) \\
& =(D-B) / B \times 100 \\
& \text { grafting efficiency, GE }(\%) \\
& =(D-B) /(A-B) \times 100
\end{aligned}
$$

where $A, B, C$, and $D$ are the weights of the crude graft product, cellulose, the monomer charged, and the product after extraction, respectively.
The following procedure was used to isolate PAN from cellulose grafted with PAN. ${ }^{12}$ First, a given sample was oxidized by immersing it in aqueous potassium periodate at room temperature for $6 \mathrm{~h}$. Then the reaction mixture was filtered to collect the residue, which was immersed in $2.5 \mathrm{~N}$ sodium hydroxide at room temperature for $24 \mathrm{~h}$. The remaining residue was obtained by filtration, dried, and dissolved in DMF. After filtration, PAN was recovered from the filtrate by precipitating into methanol.

Cellulose grafted with PMMA was hydrolyzed with $72 \% \mathrm{H}_{2} \mathrm{SO}_{4}$ to isolate PMMA. ${ }^{13}$

Intrinsic viscosities, $[\eta]$ (in $\mathrm{cm}^{3} \mathrm{~g}^{-1}$ ), of the isolated graft polymers were measured in a Ubbelohde dilution viscometer at $25^{\circ} \mathrm{C}$. The solvents were DMF for PAN and acetone for PMMA and were used to estimate viscosity-average molecular weights by the following equations ${ }^{14,15}$ :

$$
\begin{aligned}
{[\eta]_{\mathrm{DMF}} } & =24.3 \times 10^{-3} \mathrm{M}^{0.75} \\
{[\eta]_{\text {acetone }} } & =5.3 \times 10^{-3} \mathrm{M}^{0.73}
\end{aligned}
$$

Graft products subjected to extraction were analyzed by thin layer chromatography in order to detect any remaining homopolymer. A stock solution was prepared by dissolving a given product in DMSO-PF according to the method described above and an aliquot was applied on a Yamato replate (Yamato Scientific). A mixture of DMF and acetone ( $4: 5$ by weight) was used as the developer. After development, chromatograms were stained with iodine vapor. In no case were any spots found associated with the homopolymer, confirming that our extraction procedure was adequate.

\section{RESULTS AND DISCUSSION}

\section{Initiation with Ammonium Persulfate}

Two peroxide initiators were tested for graft copolymerization of vinyl monomers onto cellulose. APS was found to initiate grafting in the DMSOPF system, whereas benzoyl peroxide was not effective under the same grafting conditions. The results obtained with APS are described below.

Figures 1 and 2 show the effects of polymerization temperature on the grafting of $\mathrm{AN}$ and MMA, respectively. For either monomer system, the weight conversion and the grafting percentage show maxima around $40^{\circ} \mathrm{C}$. This indicates that an 
Homogeneous Graft Copolymerization onto Cellulose

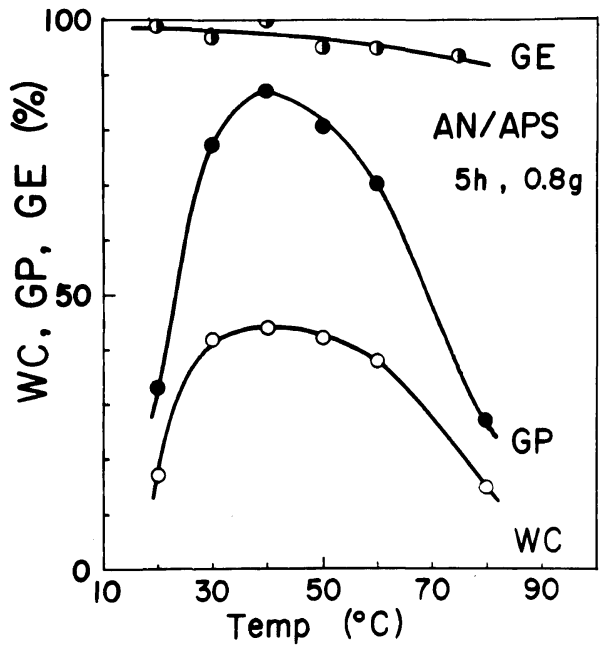

Figure 1. Effect of polymerization temperature on grafting of AN: (O) weight conversion, WC; $(\mathbf{O})$ grafting percentage, GP; () grafting efficiency, GE; cellulose, $2 \%$; AN, $4 \mathrm{~g} / 100 \mathrm{~g}$ DMSO soln; APS, $0.8 \mathrm{~g} / 100 \mathrm{~g}$ DMSO soln; grafting time, $5 \mathrm{~h}$.

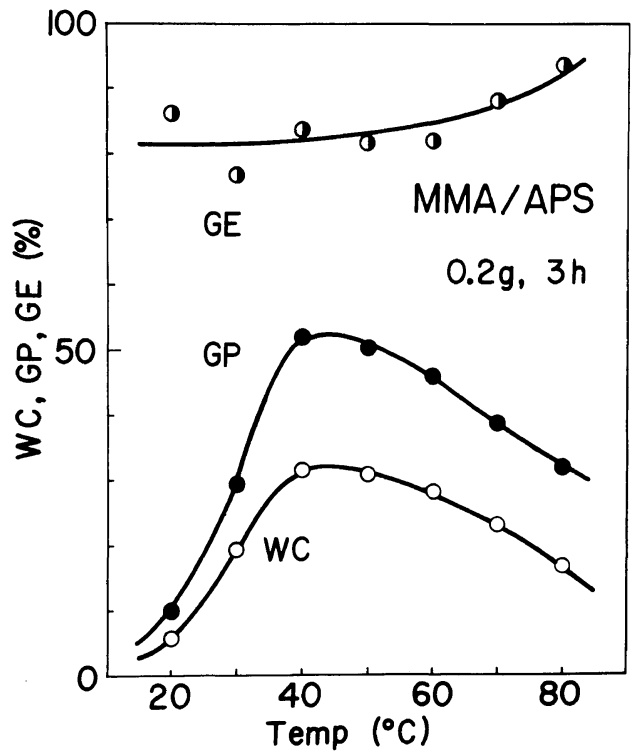

Figure 2. Effect of polymerization temperature on grafting of MMA: cellulose, $2 \%$; MMA, $4 \mathrm{~g} / 100 \mathrm{~g}$; APS, $0.2 \mathrm{~g} / 100 \mathrm{~g}$.

optimum temperature is present for each monomer system. It is also seen in these figures that for both AN and MMA, the grafting efficiency is very high and almost independent of temperature. Thus, homopolymerization scarcely took place under

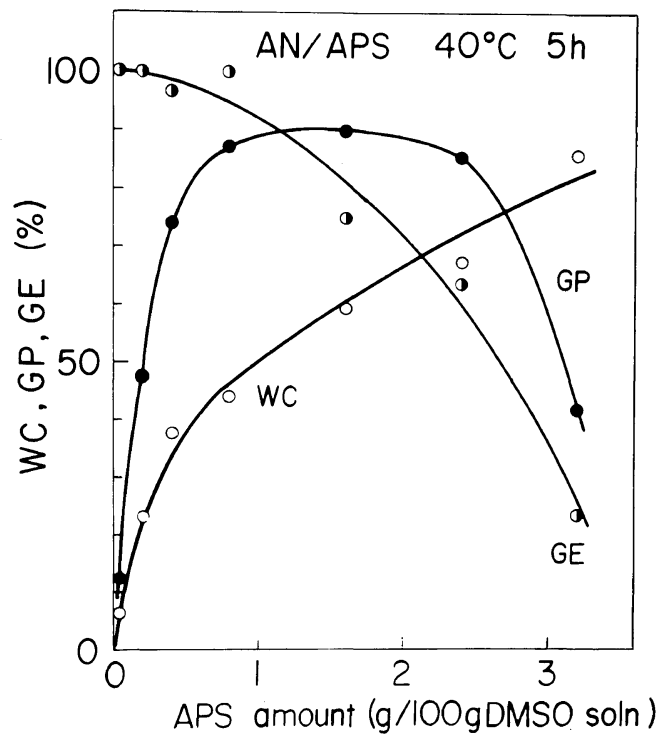

Figure 3. Effect of initiator concentration on grafting of AN: cellulose, $2 \%$; $\mathrm{AN}, 4 \mathrm{~g} / 100 \mathrm{~g}$; temp, $40^{\circ} \mathrm{C}$.

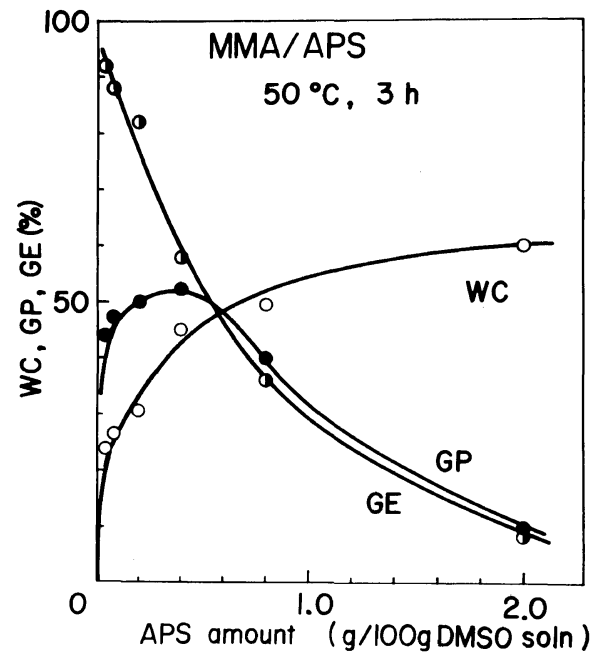

Figure 4. Effect of initiator concentration on grafting of MMA: cellulose, $2 \%$; MMA, $4 \mathrm{~g} / 100 \mathrm{~g}$.

the grafting conditions employed.

Figure 3 illustrates how the grafting of AN was affected by initiator concentration. The initiator concentration is expressed in grams of APS in $100 \mathrm{~g}$ of the DMSO solution and all the experiments were carried out at $40^{\circ} \mathrm{C}$. As the initiator concentration is increased, the weight conversion increases monotonically and the grafting efficiency decreases gradually, whereas the grafting percentage shows a 


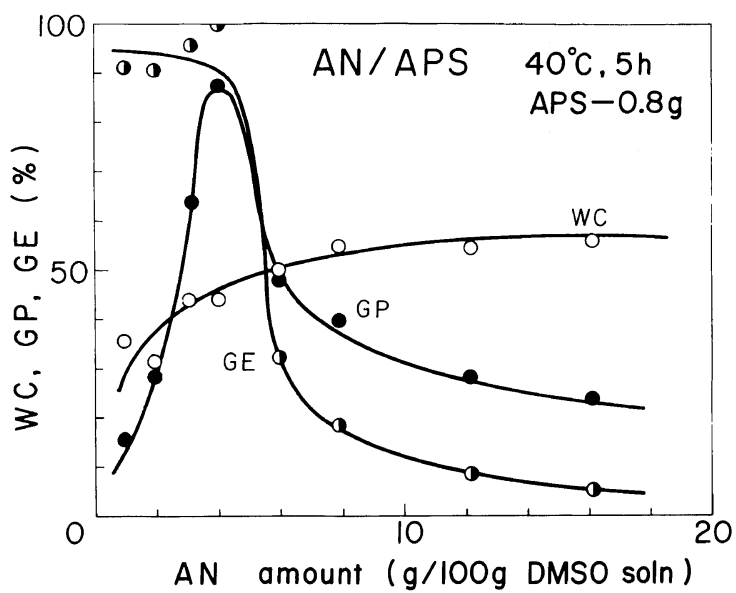

Figure 5. Effect of monomer concentration on grafting of $\mathrm{AN}$ : cellulose, $2 \%$.

broad plateau over the range of concentration between 0.8 and $2.4 \mathrm{~g} / 100 \mathrm{~g}$. The decreases in grafting percentage and grafting efficiency at higher initiator concentrations may be due to termination with primary radicals of either cellulose macroradicals or growing grafted chains or both.

Figure 4 shows similar data for the grafting of MMA. The grafting percentage increases sharply at very low APS concentrations and attains a maximum at about $0.4 \mathrm{~g} / 100 \mathrm{~g}$. On the other hand, the grafting efficiency drops rapidly with increasing APS concentration. Comparison of Figure 4 with Figure 3 shows that the range of initiator concentration effective for grafting differs greatly for the two monomers. Further, the maximum grafting percentage of AN is larger than that of MMA.

It should be noted that an opposite trend was found for grafting onto cellulose in a heterogeneous system. ${ }^{16}$ The lower grafting percentage of $\mathrm{AN}$ is ascribed to precipitation of PAN on cellulose fibers. Thus we find that the difference in the grafting method gives rise to very different results.

Figure 5 shows the results from experiments performed for finding the effect of monomer concentration on the grafting of AN. The monomer concentration is expressed in grams of the monomer in $100 \mathrm{~g}$ of the DMSO solution. The initiator concentration was chosen to be $0.8 \mathrm{~g} / 100 \mathrm{~g}$ which is, as seen from Figure 3, the optimum condition with respect to the grafting percentage and the grafting efficiency at a fixed monomer concentration of $4 \mathrm{~g}$ / $100 \mathrm{~g}$.

As the monomer concentration is increased, the grafting percentage passes through a steep maximum at about $4 \mathrm{~g} / 100 \mathrm{~g}$, whereas the grafting efficiency suddenly decreases at nearly the same concentration and gradually at higher concentrations. The weight conversion stays virtually constant over the concentration range examined. The decrease in concentration of monomer, which is a nonsolvent for cellulose, would lower the solubility of cellulose in the DMSO-PF solvent system, thus explaining the decreases in grafting percentage and grafting efficiency at higher AN concentrations.

Similar results were obtained for the grafting of MMA as shown in Figure 6. The grafting percentage exhibits a maximum at about $4 \mathrm{~g} / 100 \mathrm{~g}$.

It was not possible to perform grafting experiments at fixed initiator and monomer concentrations and varying cellulose concentration. This was because a DMSO solution of cellulose is very viscous and the highest attainable concentration for the cellulose sample used was about $4 \mathrm{wt} \%$. Alternatively, we performed grafting experiments in which the monomer concentration was varied, with the molar ratio of monomer to initiator, $[\mathrm{M}] /[\mathrm{I}]$, kept constant. The values of $[\mathrm{M}] /[\mathrm{I}]$ were 22 and 46 for AN and MMA, respectively. These values correspond to the optimum conditions at a fixed cellulose concentration of $2 \%$.

Figures 7 and 8 show the results for $\mathrm{AN}$ and MMA, respectively. It should be noted here that the increase in monomer amount corresponds to the decrease in cellulose concentration. With either monomer, the weight conversion increases gradually with increasing monomer concentration, 
Homogeneous Graft Copolymerization onto Cellulose

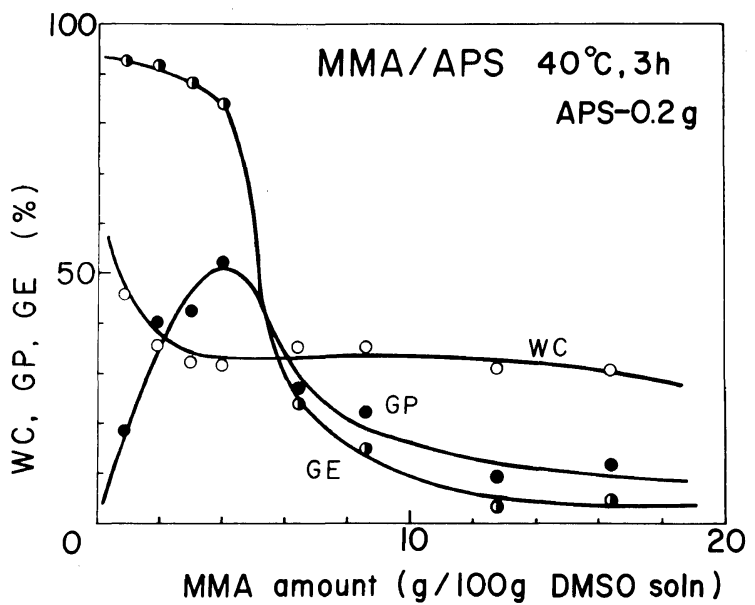

Figure 6. Effect of monomer concentration on grafting of MMA: cellulose, $2 \%$.

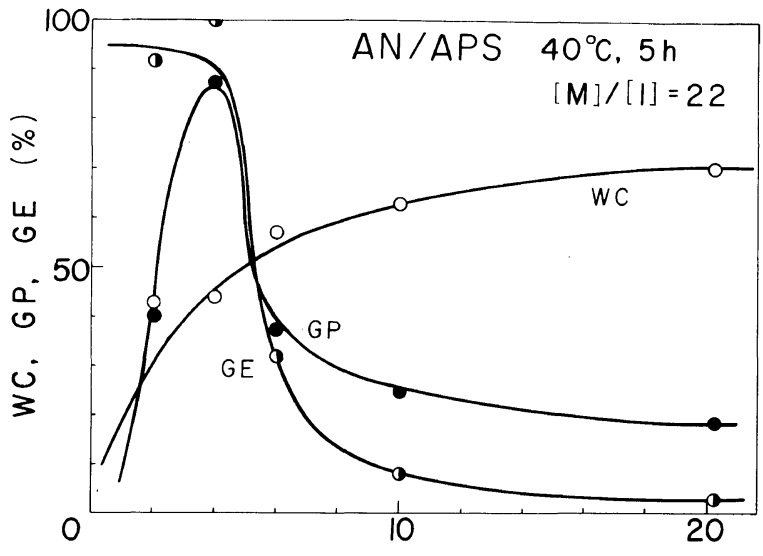

AN amount ( $g / 100 \mathrm{~g}$ DMSO soln )

Figure 7. Effect of monomer concentration on grafting of $\mathrm{AN}$, with molar ratio of monomer to initiator, $[\mathrm{M}] /[\mathrm{I}]$, kept constant: cellulose, $2 \%$.

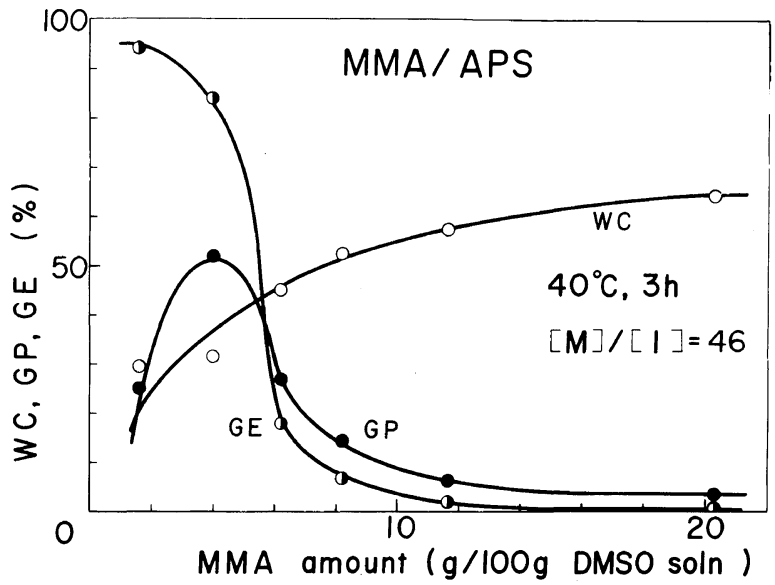

Figure 8. Effect of monomer concentration on grafting of MMA, with molar ratio of monomer to initiator kept constant: cellulose, $2 \%$. 
while the grafting percentage shows a maximum around $4 \mathrm{~g} / 100 \mathrm{~g}$ and the grafting efficiency drops sharply at a concentration slightly higher than this. This result with grafting efficiency is consistent with the well-known fact that the grafting efficiency increases with increasing polymer concentration.

\section{Initiation with Azobisisobutyronitrile}

It is well known that the nature of the initiator has a profound effect on grafting. For example, AIBN is known to show resonance stabilization. No such resonance stabilization exists in peroxide initiators. These are the reasons for the generally accepted argument that a higher grafting yield would be obtained with peroxide initiators than with AIBN. However, in our grafting experiments with vinyl monomers onto 6-nylon in $\mathrm{CaCl}_{2}$-methanol solvent system, AIBN was found to be more effective than peroxide initiators. ${ }^{17,18}$ A similar result has been reported for the grafting of vinyl acetate onto cellulose in a heterogeneous system. ${ }^{19}$

Grafting in the DMSO-PF system was also performed with AIBN as the initiator. Figure 9 shows the temperature dependence of the three parameters for AN. Although the weight conversion increases remarkably with raising temperature, both the

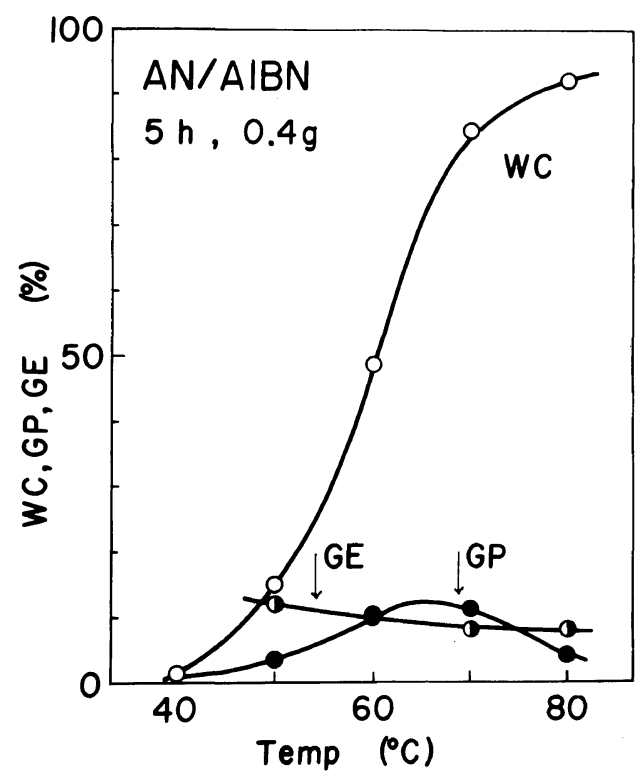

Figure 9. Effect of polymerization temperature on grafting of $\mathrm{AN}$ : cellulose, $2 \% ; \mathrm{AN}, 4 \mathrm{~g} / 100 \mathrm{~g} ; \mathrm{AIBN}$, $0.4 \mathrm{~g} / 100 \mathrm{~g}$. grafting percentage and the grafting efficiency are $10 \%$ at most. No higher values were obtained for the latter parameters, even though the concentrations of initiator, monomer, and cellulose were varied as much as possible. Since the chain transfer from AIBN primary radicals may be negligible due to resonance stabilization, we conclude that the chain transfer from the PAN homopolymer radicals to cellulose scarcely took place in this monomer-

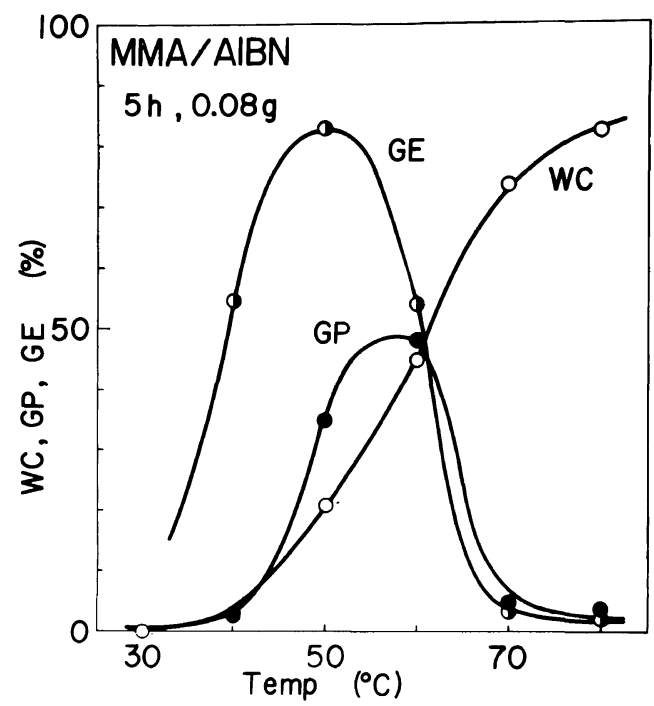

Figure 10. Effect of polymerization temperature on grafting of MMA: cellulose, $2 \%$; MMA, $4 \mathrm{~g} / 100 \mathrm{~g}$; AIBN, $0.08 \mathrm{~g} / 100 \mathrm{~g}$.

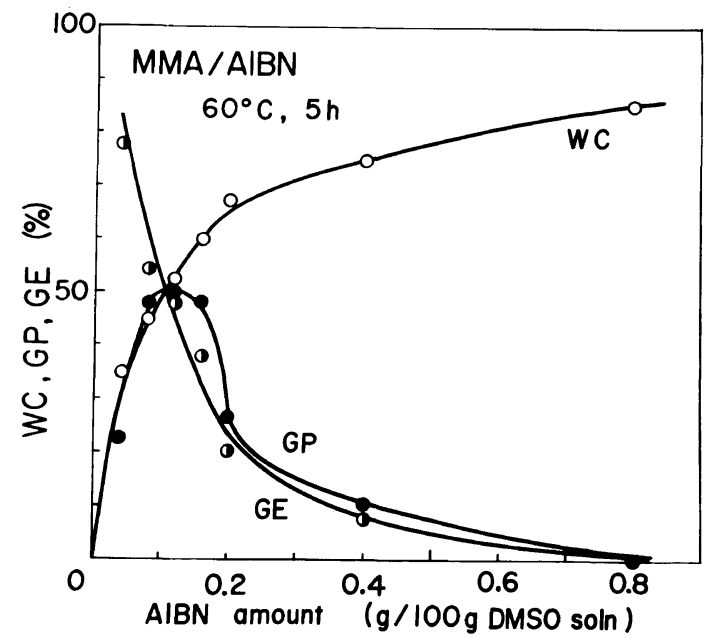

Figure 11. Effect of initiator concentration on grafting of MMA: cellulose, $2 \%$; MMA, $4 \mathrm{~g} / 100 \mathrm{~g}$.

Polymer J., Vol. 13, No. 12, 1981 


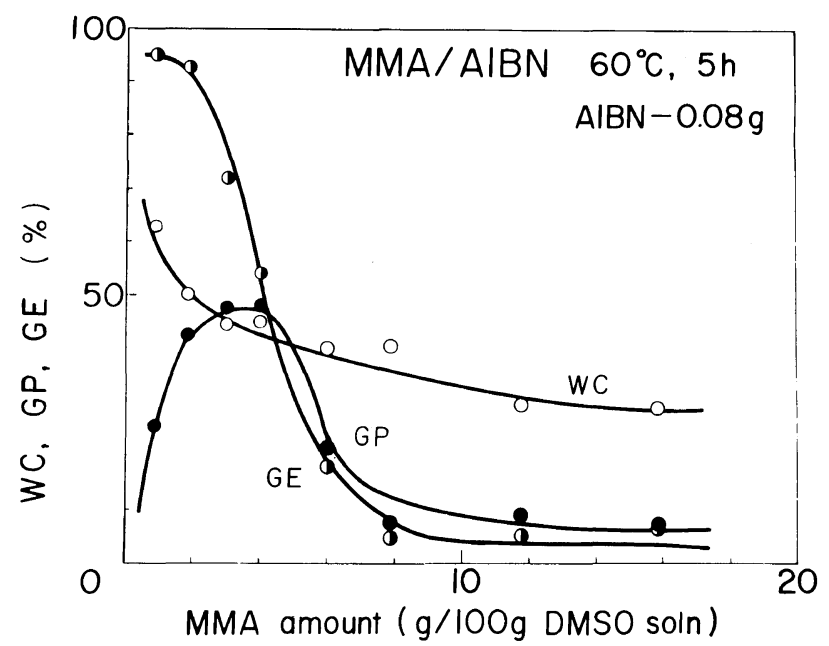

Figure 12. Effect of monomer concentration on grafting of MMA: cellulose, $2 \%$.

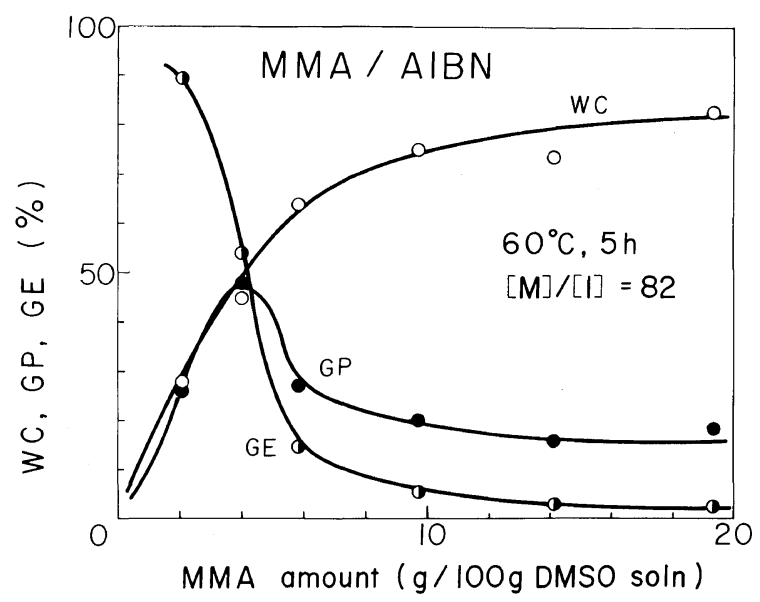

Figure 13. Effect of monomer concentration on grafting of MMA, with molar ratio of monomer to initiator kept constant: cellulose, $2 \%$.

initiator system.

The effect of temperature on the grafting of MMA is shown in Figure 10. In contrast with the case of $\mathrm{AN}$, both the grafting percentage and the grafting efficiency exhibit maxima around $50^{\circ} \mathrm{C}$. This clearly indicates that grafting took place effectively with MMA. The difference between the two monomers may be attributed to the higher reactivity of PMMA homopolymer radicals than that of PAN radicals, which would enable the former radicals to produce cellulose macroradicals more easily.

Figure 11 shows the effect of the initiator concentration on the grafting of MMA. Comparison of this figure with Figure 4 reveals that the maximum grafting percentage attained in each initiator system is almost the same, whereas the effective concentration range of initiator is much lower and narrower in the AIBN system than in the APS system. As mentioned above, cellulose macroradicals must be formed primarily by the attack of homopolymer radicals in the AIBN system. On the other hand, in the APS system, both PMMA homopolymer radicals and APS primary radicals participate in forming cellulose macroradicals. This combined action of the two types of radicals is expected to widen the effective concentration range of the 
Table I. Characterization of graft copolymers ${ }^{a}$

\begin{tabular}{clcccc}
\hline Monomer & Initiator & {$[\mathrm{M}] /[\mathrm{I}]$} & $\begin{array}{c}\text { Grafting } \\
\text { percentage }\end{array}$ & $\begin{array}{c}M_{v} \text { of grafts } \\
\times 10^{-4}\end{array}$ & $\begin{array}{c}\text { Number } \\
\text { of grafts }\end{array}$ \\
\hline \multirow{2}{*}{ AN } & APS & 22 & 87.3 & 3.6 & 3.9 \\
& AIBN & 31 & 10.0 & 3.4 & 0.5 \\
& APS & 46 & 52.0 & 2.5 & 3.4 \\
& AIBN & 82 & 48.0 & 6.0 & 1.3 \\
\hline
\end{tabular}

$M_{v}$ of cellulose $=16 \times 10^{4}$

a Grafting condition: cellulose concn, $2 \mathrm{wt} \%$; monomer concn, $4 \mathrm{~g} / 100 \mathrm{~g}$ DMSO soln. All other parameters for each monomer-initiator system are shown in Figures 7, 9, 8, and 13, respectively.

initiator in the APS system.

Figures 12 and 13 illustrate how the grafting of MMA is affected by the concentrations of monomer and cellulose, respectively. The features of these figures are essentially the same as those of Figures 6 and 8 , respectively. This implies that the concentration of cellulose macroradicals for each initiator system is the same even though the initiator concentration differs.

\section{Characterization of Graft Copolymers}

Physical and chemical properties of a graft copolymer should depend on such molecular parameters as the molecular weight of backbone cellulose, the number of grafts per backbone chain and their average molecular weight, etc. Thus, these parameters were determined with selected samples. The molecular weight of the backbone cellulose was estimated to be $16 \times 10^{4}$ from $[\eta]$ obtained with the DMSO-PF solvent at $30^{\circ} \mathrm{C}^{20}$ and with the cadoxen solvent at $20^{\circ} \mathrm{C}^{21}$ Some of the graft copolymers obtained under the optimum conditions for each monomer-initiator system were hydrolyzed to isolate graft polymers and their molecular weights were estimated from $[\eta]$. The results are given in Table I.

It should be noted from this table that the number of grafts per cellulose chain is relatively large in either monomer-initiator system. This is probably because, in the homogeneous system employed here, all the cellulose chains are exposed to chain transfer reactions leading to the formation of cellulose macroradicals. This is in contrast to heterogeneous systems in which grafting occurs only in amorphous regions, causing the number of grafts per cellulose chain seldom to exceed unity. ${ }^{3-5,22,23}$
Our data may be compared with the homogeneous grafting of styrene onto cellulose in a sulfur dioxide-diethylamine-DMSO mixture, ${ }^{7}$ which resulted in the number of grafts as large as $2.4-10.6$.

Although the molecular weights of PAN grafts obtained were nearly the same, the number of grafts was much larger for the APS system than for the AIBN system. This difference between the two initiators may be accounted for by the difference in the mechanism of cellulose macroradical formation discussed in relation to Figure 11.

The number of grafts for the MMA-AIBN system is larger than unity and that for the AN-AIBN system. This result is consistent with the supposition that the chain transfer reactivity of PMMA radicals to cellulose is higher than that of PAN radicals.

\section{REFERENCES}

1. Y. Ogiwara and H. Kubota, Kogyo Kagaku Zasshi, 71, 171 (1968).

2. O. Y. Mansour and A. Nagaty, J. Appl. Polym. Sci., 23, 2425 (1979).

3. Y. Ogiwara, H. Kubota, and K. Arai, J. Appl. Polym. Sci., 14, 1049 (1970).

4. P. Lepoutre and S. H. Hui, J. Appl. Polym. Sci., 19, 1257 (1975).

5. A. Moze and S. Lapanje, Makromol. Chem., 180, 1599 (1979).

6. T. I. Min and H. Inagaki, Polymer, 21, 309 (1980).

7. M. Tsuzuki, I. Hagiwara, N. Shiraishi, and T. Yokota, J. Appl. Polym. Sci., 25, 2909 (1980).

8. K. Suzuki and I. Ikeda, Polym. Prepr., Jpn., 29, 146 (1980).

9. M. Tsuzuki, I. Hagiwara, N. Shiraishi, and T. Yokota, J. Appl. Polym. Sci., 25, 2721 (1980).

10. S. M. Hudson and J. A. Cuculo, J. Macromol. Sci., Rev. Macromol. Chem., C18, 1 (1980).

11. D. C. Johnson, M. D. Nicholson, and F. C. Haigh, 
Appl. Polym. Symp., No. 28, 931 (1976).

12. P. Cremonest, Cellulose Chem. Technol., 2, 459 (1968).

13. F. Ide, Kogyo Kagaku Zasshi, 64, 1489 (1961).

14. R. L. Cleland and W. H. Stockmayer, J. Polym. Sci., 17, 473 (1955).

15. H.-J. Cantow and G. V. Schulz, Z. Physik. Chem., 2, 117 (1954).

16. F. Ide, Kogyo Kagaku Zasshi, 65, 82 (1962).

17. K. Kosai, T. Higashino, and S. Ohue, Nippon Kagaku Kaishi, 523 (1975).
18. K. Kosai, T. Higashino, and N. Nishioka, Nippon Kagaku Kaishi, 561 (1977).

19. B. M. Misra, J. K. Jassal, and C. S. Pande, J. Polym. Sci., Polym. Chem. Ed., 16, 295 (1978).

20. H. A. Swenson, Appl. Polym. Symp., No. 28, 945 (1976).

21. G. Jayme and P. Kleppe, Papier, 15, 492 (1961).

22. I. Sakurada, Y. Ikada, and Y. Nishizaki, J. Polym. Sci., C, No. 37, 265 (1972).

23. T. Taga and H. Inagaki, Angew. Makromol. Chem., 23, 129 (1973). 